\title{
Perfil sistémico-funcional del grupo nominal en español: estructura, funciones discursivas básicas y organización sistémica $^{1}$
}

\section{A systemic-functional profile of the nominal group in Spanish: structure, basic discursive functions and systemic organisation}

\author{
BEATRIZ QUIROZ OLIVARES ${ }^{\mathrm{a} 2}$ \\ JAMES MARTIN ${ }^{\mathrm{a}, \mathrm{b} 3}$ \\ a Pontificia Universidad Católica de Chile, Chile. \\ Correo electrónico: bquiroz@uc.cl \\ ${ }^{b}$ The University of Sydney, Australia.
}

Este artículo propone una descripción sistémico-funcional del grupo nominal en español basada en textos situados. Primero, la estructura del grupo nominal se aborda 'por arriba' desde las variables contextuales de campo y de modo para introducir dos sistemas semántico-discursivos claves, el de idEACión, y de idENTIFICACión. Luego se describen las funciones estructurales que, en la lexicogramática, están al servicio de la IDEACión, incluidas las funciones Cosa, Clasificador y Epíteto; luego se centra la atención en las funciones Deíctico y Cualificador, que realizan opciones en IDENTIFICACión. Sobre la base de las funciones descritas para la estructura, junto con las clases de unidades que las realizan en la escala de rango, se propone un perfil sistémico del grupo nominal en español, con las distintas opciones en juego. Finalmente se revisan las implicancias del enfoque adoptado para un trabajo descriptivo y aplicado de orientación funcional.

Palabras clave: grupo nominal, español, gramática sistémico-funcional, semántica del discurso, redes sistémicas.

\footnotetext{
${ }^{1}$ El trabajo presentado en este artículo ha recibido el apoyo de la Vicerrectoría Académica de la Pontificia Universidad Católica de Chile, por medio del proyecto FRCAI1720, y de ANID-FONDECYT, por medio del proyecto $\mathrm{N}^{\circ} 11170674$. Los autores agradecen los valiosos comentarios de los evaluadores anónimos, que han permitido clarificar, precisar y mejorar el contenido de esta versión final. Todos los errores y omisiones que persisten son de exclusiva responsabilidad de los autores.

${ }^{2}$ ORCID: https://orcid.org/0000-0002-6386-3837

${ }^{3}$ ORCID: https://orcid.org/0000-0002-8463-4849
} 
This paper proposes a text-based systemic-functional account of the Spanish nominal group. Firstly, approaching the Spanish nominal group 'from above' (i.e. taking the contextual variables of field and mode as a starting point), two key discourse semantic systems are introduced - IDEATION and IDENTIFICATION. The paper then describes the structural resources that contribute to the realisation of choices in IDEATION in lexicogrammar - including the Thing, the Classifier and the Epithet functions. It then moves on to the Deictic and the Qualifier functions, which realise choices in IDENTIFICATION. Based on the structural configurations described, including the classes of units realising them along the rank scale, the paper proposes a systemic profile of the Spanish nominal group, canvassing the various choices in play. Finally, the paper reviews the implications of the approach proposed for functionally-oriented descriptive and applied research.

Key words: nominal group, Spanish, Systemic Functional Grammar, discourse-semantics, system networks.

\section{INTRODUCCIÓN}

En los estudios descriptivos y aplicados inspirados por la Lingüística Sistémico Funcional (en adelante, LSF), el grupo nominal ha demostrado ser un recurso lexicogramatical importante para comprender formas de organización lingüística motivadas por funciones contextuales. Así, es clave para comprender la naturaleza del lenguaje empleado en distintos registros, p. ej., como recurso para distinguir campos especializados (disciplinares, técnicos, académicos, etc.) de los no especializados (de la vida cotidiana); también para establecer rasgos propios del modo escrito en contraste con los del modo oral. Asimismo, se trata de un recurso involucrado de manera relevante en la organización cohesiva de textos orales y escritos, tanto por su papel en la formación de cadenas léxicas como por su lugar en la formación de cadenas referenciales.

No obstante, las descripciones sistemáticas del grupo nominal en lenguas distintas del inglés (p. ej. Fontaine 2017; Halliday y Matthiessen 2014) son más bien escasas. Ha habido intentos descriptivos de orientación sistémico-funcional en portugués de Brasil (Figueredo et al. 2008), y algún intento de abordar el grupo nominal en español peninsular, pero desde una perspectiva más bien tradicional (Lavid et al. 2010). Más allá de estos y otros trabajos de orientación más aplicada, no hay de momento estudios que aborden el grupo nominal en español tomando en cuenta de manera sistemática e integrada principios fundamentales de la LSF, representados por las dimensiones de metafunción, estratificación, eje, escala de rango e instanciación (Caffarel et al. 2004; Matthiessen 2015). La falta de descripciones que tomen en cuenta estos principios plantea el problema de que, sobre todo en trabajos aplicados, se tiende a adaptar lo descrito para el inglés de manera más bien ad hoc, o bien se fuerzan, también de manera ad hoc, categorías que no han surgido de descripciones del español funcionalmente orientadas.

En este artículo nos proponemos ofrecer un perfil integrado del grupo nominal en español que tenga en cuenta la interacción entre estas dimensiones teóricas claves de la 
$\mathrm{LSF}^{4}$. En la sección 2, abordamos el grupo nominal 'por arriba', es decir, en función de dos sistemas semántico-discursivos claves (Martin 1992; Martin y Rose 2007, 2008): el sistema de IDEACIÓN, que dentro de la metafunción ideacional, define opciones globales relevantes para la variable contextual de campo; y el sistema de IDENTIFICACIÓN, que dentro de la metafunción textual define opciones globales relevantes para la variable contextual de modo. Ya desde la lexicogramática, en la sección 3 revisamos paso a paso los recursos estructurales básicos del grupo nominal en español, tomando ejemplos de un texto periodístico relativamente extenso en español de Chile. Primero nos centramos en las funciones estructurales que están al servicio de la IDEACIÓN, es decir, que contribuyen a construir ideacionalmente las entidades y las propiedades asignadas a ellas en el discurso; luego fijamos nuestra atención en las funciones estructurales que contribuyen a la IDENTIFICACIÓN, es decir, que están al servicio de la presentación y recuperación de entidades en tanto referentes discursivos. Sobre la base de las funciones estructurales descritas, en la sección 4 proponemos un perfil sistémico del grupo nominal en español, con las distintas opciones que están en juego. Finalmente concluimos el artículo abordando las implicancias del enfoque adoptado para un trabajo descriptivo y aplicado de orientación funcional.

\section{El GRUPO NOMINAL DESDE UNA PERSPECTIVA DISCURSIVA}

En este artículo se abordará el grupo nominal primero desde una perspectiva ideacional y luego desde una perspectiva textual. En términos de la variable de campo, que proporciona el contexto ideacional, los grupos nominales construyen una perspectiva estática sobre los fenómenos. En otras palabras, proporcionan recursos para establecer relaciones de clasificación y composición entre los ítems que conforman un campo dado, y(o) para asociar estos ítems a ciertas propiedades. Esta concepción del campo, basada en la propuesta de Doran y Martin (2021), se presenta en la red sistémica de la Figura 1:

Figura 1. Opciones de campo (Doran y Martin 2021).

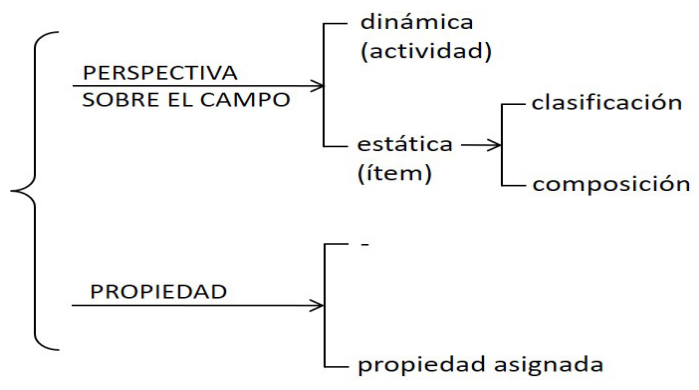

\footnotetext{
${ }^{4}$ Véase Matthiessen y Halliday (2009) (y Quiroz (2016), en español) para las convenciones sistémico-funcionales empleadas en este artículo, incluido el uso de versalitas para los nombres de sistemas, de mayúscula inicial para las funciones estructurales y de minúsculas para las clases y rasgos sistémicos.
} 
Desde la perspectiva del estrato semántico-discursivo, es decir, en términos de IDEACIÓN, los grupos nominales realizan entidades que cumplen una variedad de roles, dentro y fuera de distintos tipos de figuras (Hao 2020). En un campo, las entidades semánticodiscursivas realizan, la mayor de las veces, ítems - personas, lugares y cosas (incluidas entre estas últimas tanto objetos como instituciones). Las entidades también pueden designar actividades, mediante grupos nominales que contienen sustantivos analizados normalmente como 'nombres eventivos', p. ej., proceso, viaje, travesía, periplo, peregrinaje, expedición (entidades de actividad); pueden, asimismo, corresponder a entidades que representan discurso, p. ej. por medio de grupos nominales con sustantivos como anécdota, broma, acertijo, ensayo, párrafo, capitulo, indice, argumento, factor, pregunta (entidades semióticas). Una versión simplificada de las opciones propuestas por Hao (2020) para el sistema de ENTIDAD (dentro del sistema mayor de IDEACIÓN) se presenta en la Figura 2:

Figura 2. Red simplificada para tipos de entidades semántico-discursivas (Hao 2020)5.

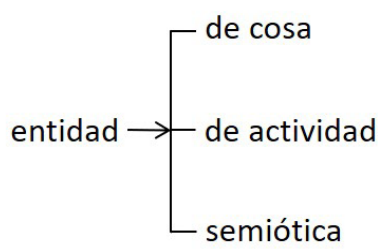

En el estrato lexicogramatical, la función clave involucrada en la realización de entidades semántico-discursivas en la estructura del grupo nominal es la función Cosa (Thing). Esta función puede ser suficiente por sí sola para realizar una entidad, como ocurre cuando el grupo nominal contiene un pronombre (ellas) o un nombre propio (Santiago) como núcleo. Los grupos nominales, sin embargo, pueden ser bastante más complejos, dependiendo de las funciones semántico-discursivas ideacionales que deban cumplir en un texto.

Parte de esta complejidad está también condicionada por la variable registral de modo, que proporciona el contexto a la metafunción textual. Como se ha planteado en la literatura (p. ej. Halliday 1989, 1998; Martin 1993a, 1993b), cuando el lenguaje tiende a cumplir una función auxiliar dentro de lo que sucede en el contexto de situación (p. ej. en la escasa interacción verbal que ocurre entre los jugadores de un partido de fútbol), la contribución de los grupos nominales será primordialmente la de recuperar referentes dentro y fuera del texto. En este tipo de contextos en español, de hecho, tienden a estar en

\footnotetext{
${ }^{5}$ Hao (2020: 64) también incluye como opciones primarias a las entidades fuente (source entities), las entidades de tiempo (time entities) y las entidades de lugar (place entities), además de ofrecer criterios gramaticales para distinguir entre todas ellas (y sus subtipos) en el ámbito del grupo nominal (inglés).
} 
juego recursos que caben más bien dentro del ámbito del grupo verbal, como los clíticos pronominales ( $m e$, la, les, nos, etc.) y las distinciones de persona en la flexión verbal (Quiroz 2017b), que recuperan entidades en el discurso sin necesidad de recurrir a grupos nominales. En cambio, en contextos de situación donde lo que sucede tiene primordialmente que ver con el uso del lenguaje (p. ej. relatar una anécdota, escribir un artículo), los grupos nominales se ven mucho más exigidos en su estructuración y, por tanto, en los recursos que despliegan para introducir referentes y recuperarlos a lo largo del texto. Desde un punto de vista semántico-discursivo, estas variaciones en el modo inciden en la selección de las opciones disponibles en el sistema de idENTIFICACión, dentro de la metafunción textual (Martin 1983, 1992; Martin y Rose 2007).

\section{EL GRUPO NOMINAL EN DOS FUNCIONES DISCURSIVAS: RECURSOS IDEACIONALES Y TEXTUALES}

En esta sección se exploran con detenimiento los principales recursos estructurales del grupo nominal en español, siempre tomando en cuenta las motivaciones semánticodiscursivas que hemos descrito en la sección anterior. En primer lugar, revisaremos los recursos que contribuyen a la metafunción ideacional y que, por tanto, interactúan de manera relevante con opciones discursivas en el sistema de IDEACIÓN (variable registral de campo). En segundo lugar, revisaremos los recursos que contribuyen a la metafunción textual y que, por tanto, están al servicio de las opciones discursivas del sistema de IDENTIFICACión (variable registral de modo). Las generalizaciones que presentamos aquí son el resultado del análisis cualitativo del corpus más amplio del proyecto ANID-FONDECYT 11170674, conformado por 367 textos orales y escritos de español de Chile, provenientes de distintos géneros y registros (224.887 palabras en total). Con el fin de mostrar de un modo más claro las distintas contribuciones discursivas del grupo nominal, en este artículo emplearemos primordialmente ejemplos de uno de estos textos, el reportaje 'La ola maldita' de Guzmán (2010). Este reportaje recoge los testimonios de habitantes de Constitución y su experiencia durante y tras el tsunami que arrasó a esta ciudad chilena el 27 de febrero de 2010. Optamos por emplear este texto porque articula en su interior, en tanto macrogénero, una serie de relatos (stories) (Martin y Rose 2008). Los relatos tienden a ofrecer grupos nominales muy variados, puesto que realizan una gran variedad de entidades discursivas. Estas entidades no solo son introducidas y recuperadas de diversas maneras a lo largo del reportaje, sino que también son cuantificadas, descritas y clasificadas de diversas maneras dentro del campo que se va construyendo.

\subsection{Recursos ideacionales del grupo nominal}

Esta subsección se centra en los grupos nominales en los que la función Cosa es realizada por sustantivos comunes. La realización de esta función mediante pronombres y sustantivos propios se abordará en la sección 3.2. 
Desde la perspectiva del campo, las principales taxonomías construidas en el texto de Guzmán remiten al tsunami y sus efectos en las personas y su entorno. En el siguiente extracto textual del reportaje, los sustantivos comunes que realizan la función Cosa aparecen en negrita:

Los jóvenes entraron al hospital sin esperanza. Era un caos de heridos y muertos. No creían que nadie pudiera sobrevivir a la ola que habian visto, pero en la lista de ingresados, el nombre de Gabriel les saltó en la cara.

Jonathan entró a la carrera a la zona de los pacientes y recorrió las camillas hasta que lo encontró. Le pegó tres garabatos y lo abrazó. Gabriel sólo tenía heridas en los pies. Tuvo la fortuna de que la ola lo llevara directo a la orilla.

Los grupos nominales que solo contienen una Cosa (esperanza, heridos, muertos, heridas) se analizarán como se muestra en (1), es decir, distinguiendo claramente entre funciones y clases, según los supuestos sistémico-funcionales de organización de la escala de rango:

(1)

esperanza
\begin{tabular}{|l|l|}
\hline grupo nominal & clase de grupo \\
\hline Cosa & función de grupo \\
\hline sust. común & clase de palabra \\
\hline
\end{tabular}

En español, en contraste con lo que puede verse en las descripciones del grupo nominal en inglés (Halliday y Matthiessen 2014), los grupos nominales que solamente contienen un sustantivo (también denominados 'sintagmas nominales escuetos') son infrecuentes y aparecen en entornos bien acotados. En términos generales, sin embargo, vemos en el extracto anterior que la función Cosa es en varios casos suficiente para delimitar ideacionalmente ('léxicamente') a la entidad que se está construyendo en el campo: el hospital, la cara, los pacientes, las camillas, tres garabatos, los pies, la ola, la orilla.

Hay casos en los que se requiere una función adicional en la estructura, como en el bote pesquero y generador eléctrico. Esta función puede involucrar adjetivos que clasifican a la entidad que está en juego y que, en (2) y (3), asumen por tanto la función de Clasificador, inmediatamente pospuesta a la función Cosa en la secuencia:

(2)

bote pesquero
\begin{tabular}{|l|l|l|}
\hline grupo nominal & clase de grupo \\
\hline Cosa & Clasificador & función de grupo \\
\hline sust. Común & adjetivo & clase de palabra \\
\hline
\end{tabular}


(3)

generador
\begin{tabular}{|l|l|l|}
\hline grupo nominal & clécrico & clase de grupo \\
\hline Cosa & Clasificador & función de grupo \\
\hline sust. Común & adjetivo & clase de palabra \\
\hline
\end{tabular}

Dado que en (2) y (3) la función de los adjetivos es más la de clasificar que la de describir, ninguno de ellos, pesquero o eléctrico, admite, en tanto Clasificadores, graduación: * el bote muy pesquero no tiene sentido. Además, a diferencia de adjetivos que en español realizan otras funciones (que veremos más adelante), los adjetivos que realizan Clasificadores no pueden nunca anteceder a la Cosa. De esto se desprende el supuesto sistémico-funcional de que una función estructural no mantiene una relación biunívoca con una clase de unidad única. De hecho, lo inverso también es cierto: una misma función puede ser realizada por más de una clase. Desde la perspectiva funcional que se ofrece aquí, en consecuencia, los Clasificadores pueden ser realizados también por un sustantivo 'adposicional' (Suñer Grataços 1999), p. ej. hora punta o coche bomba. Este tipo de Clasificadores no son muy productivos en espańol, a diferencia de lo que ocurre con los Clasificadores del grupo nominal inglés, mayoritariamente realizados por sustantivos, aunque antepuestos a la Cosa, p. ej. 'wheel chair', 'war movies'; véase Halliday y Matthiessen 2014). Lo que sí es bastante productivo en español es la configuración en la que un sustantivo clasifica a la Cosa, pero antecedido por la partícula de, como en silla de ruedas, películas de guerra:

(4)

\begin{tabular}{|c|c|c|c|}
\hline \multicolumn{3}{|c|}{ grupo nominal } & de \\
\hline Cosa & \multicolumn{2}{|c|}{ Clasificador } & función de grupo \\
\hline sust. común & partícula & sust. común & clase de palabra \\
\hline
\end{tabular}

Fuera del marco sistémico-funcional, este tipo de configuración ha sido descrito de distintas maneras. Bartoš (1980) considera que estas construcciones 'de + sustantivo' constituyen, junto a los llamados 'adjetivos relacionales', los principales recursos para la 'atribución relacional' en español. Hernanz y Brucart (1987), por su parte, proponen que tanto las configuraciones adjetivales como las 'de + sustantivo (escueto)' constituyen más bien tipos de 'clasificadores'. Por su parte, Bosque (1993), quien trata esta construcción con de como 'complemento clasificativo', argumenta que este tipo de 'compuestos' no tienen el estatus de frase preposicionales. Aquí podríamos agregar configuraciones análogas, aunque mucho menos frecuentes, que involucran a partículas como a en estufa a gas, o con en café con leche. 
Los grupos nominales con Clasificadores adjetivales o sustantivos - incluyan o no una partícula - tienen en común el hecho de que discursivamente realizan una sola entidad, no dos. Grupos nominales como la escala, la escala de magnitud, la escala de magnitud sismológica refieren a una sola escala. Por tanto, son recuperados como un solo referente en el discurso (p. ej. por medio del clítico acusativo la o la tercera persona singular de la flexión verbal) ${ }^{6}$. Compárense, a este respecto, otros recursos que son tratados en español genéricamente como 'complementos nominales' y que sí introducen entidades discursivas adicionales:

\section{la angustia de ese anciano \\ los padres de los otros jóvenes la mente de muchos chilenos los lamentos de otras cuatro personas}

En los ejemplos, ese anciano, los otros jóvenes, muchos chilenos y otras cuatropersonas son grupos nominales con estructura propia, que se especifican y expanden de diversas maneras. Sirven de término a una preposición y, por tanto, forman parte de frases preposicionales propiamente tales. En términos semántico-discursivos, las frases preposicionales de los ejemplos no clasifican a la entidad realizada por la Cosa, sino que especifican su referencia en relación con una segunda (o más) entidades. Como se verá en la sección 3.2, estas frases preposicionales realizan en la estructura del grupo nominal la función de Cualificador.

En contraste, como señalan Hernanz y Brucart (1987), las construcciones clasificadoras con de (u otras partículas análogas) no muestran el mismo potencial que las frases preposicionales. Estas configuraciones involucran a sustantivos escuetos, lo que implica que tienen un potencial de expansión muy limitado, porque no es posible incorporar otras funciones en la estructura (i.e. en términos sistémico-funcionales, no es posible especificar el sustantivo por medio de un Deíctico, un Numerativo, un Epíteto o un Cualificador, como iremos viendo más adelante $)^{7}$. Constituyen, así, un subtipo de 'adposición restrictiva' (Suñer Grataços 1999). Por esta razón, proponemos en el ejemplo (5), tomado del corpus más amplio que enmarca este estudio, un análisis específico para

\footnotetext{
${ }^{6}$ En este sentido, cabe mencionar los distintos grados de integración ideacional ('léxica') posibles entre los elementos que aquí analizamos como dos funciones separadas, Cosa y Clasificador. Ejemplos como coche bomba y silla de ruedas podrían también analizarse como palabras compuestas y, por lo tanto, como elementos que unitariamente realizan la función Cosa. Este grado de integración tiene mucho que ver con la dimensión sistémico-funcional de rango, la que, por razones de espacio, no exploramos aquí en relación con el grupo nominal (pero véase Quiroz 2017b en relación con el grupo verbal en español y el lugar que los clíticos ocupan dentro de su ámbito).

${ }^{7}$ La ausencia de determinación explica por qué los Clasificadores muy rara vez aparecen vinculados a la función Cosa por medio de las contracciones del o al, que sí son empleadas en las frases preposicionales que realizan la función de Cualificador (p. ej. la tripulación del Pinita).
} 
los Clasificadores sustantivos con partícula (la función Deíctico, que también aparece en el ejemplo, será abordada en la sección 3.2):

(5)

La escala de magnitud $^{*}$
\begin{tabular}{|l|l|l|l|l|}
\hline grupo nominal & \multicolumn{2}{l|}{ clase de grupo } \\
\hline Deíctico & Cosa & \multicolumn{2}{|l|}{ Clasificador } & función de grupo \\
\hline artículo & sust. común & part. & sust. propio & clase de palabra \\
\hline
\end{tabular}

* Este ejemplo podría admitir como Clasificador a un sustantivo propio: escala de Mercalli. Este análisis se justificaría desde el punto de vista de la variable registral del campo, toda vez que el Clasificador es una función en la estructura del grupo nominal que contribuye de manera clave a la construcción de taxonomías especializadas (Halliday 1998; Martin 1993a, 1993b; Hao 2020). En el campo de la sismología en particular, nos remite a una taxonomía de escalas sismológicas cuyas designaciones provienen del individuo (investigador) que postuló originalmente dicha escala y que, en tanto Clasificador, ya no funciona como un referente individualizado (Cosa), sino como elemento que subcategoriza a la entidad 'escala sismológica'.

Si pasamos de la función discursiva de clasificar entidades a la de asignar cualidades a las entidades (Hao 2020), los grupos nominales del texto 'La ola maldita' despliegan otros recursos. La función estructural asociada a la asignación de cualidades es la de Epíteto, realizada primordialmente en español por medio de los llamados adjetivos 'calificativos' (Demonte 1999; Di Tullio 2012). El Epíteto aparece, la mayoría de las veces, pospuesto a la función Cosa (y a los Clasificadores presentes), como se ve en (6) y en la fase del relato de Hugo Barrera que presentamos a continuación:

(6)

Un ruido
\begin{tabular}{|l|l|l|l|}
\hline grupo nominal & clase de grupo \\
\hline Deíctico & Cosa & Epíteto & función de grupo \\
\hline artículo & sust. común & adjetivo & clase de palabra \\
\hline
\end{tabular}

Hugo Barrera la vio venir, encaramado en un eucalipto a unos siete metros de altura. Dice que era una masa café, furiosa, veloz, que arrastraba todo a su paso. Una masa que se extendia por todo el horizonte, que avanzaba en silencio y que cuando tocó la isla empezó a hacer un ruido ensordecedor, un "pac, pac, pac" siniestro e imparable que era provocado por cientos de árboles partidos como fósforos o arrancados de raiz. La ola azotó el árbol en el que Hugo estaba, lo zarandeó un rato, como si el destino aún no decidiera qué hacer con él y finalmente lo lanzó al agua. Hugo cayó a ese furioso torrente sabiendo que moriria. 
Los adjetivos que realizan Epítetos, a diferencia de los adjetivos que realizan Clasificadores, son graduables, p. ej., por medio de adverbios de grado o elativos: un 'pac, pac' pac' muy siniestro, un ruido extraordinariamente ensordecedor. Además, si bien en el reportaje de Guzmán (2010) aparecen mayoritariamente postpuestos a la Cosa, los Epítetos también pueden aparecer antepuestos, como en ese furioso torrente. Nótese que los Epítetos pueden aparecer pospuestos a un Clasificador y, por la estrecha relación entre Cosa y Clasificador para la realización de entidades discursivas, no puede interpolarse entre ellos (como en general, tampoco puede hacerlo ninguna otra función). En (7), se muestran las posibilidades de posición para el Epíteto eficaz, destacado en negrita:
a. Una escala sismológica eficaz
b. *Una escala eficaz sismológica
c. Una eficaz escala sismológica.

Al parecer, son diversos los factores que inciden en la posición de los Epítetos adjetivales en español. No obstante, como señala Di Tullio (2012), la posición prenominal parece restringirse más claramente a adjetivos adverbiales y a adjetivos calificativos 'evaluativos'. Es posible así notar al menos dos cosas: por una parte, la posibilidad de movimiento de los adjetivos que funcionan como Epítetos claramente los distingue de los adjetivos que funcionan como Clasificador (que no pueden anteponerse en ningún contexto) y, por otra parte, los Epítetos antepuestos parecen estar asociados más claramente a distinciones de carácter interpersonal, particularmente en términos de GRADACión y ACTiTuD (Martin y White 2005). En el cuadro 1 presentamos ejemplos de ambas posiciones, tomados del texto que estamos analizando:

Cuadro 1. Posición de la función Epíteto

\begin{tabular}{|l|l|}
\hline Epíteto $\wedge$ Cosa & Cosa ^ Epíteto \\
\hline la peor ola & esa pared oscura \\
\hline una nueva montaña & una isla inundada \\
\hline un buen rato & una velocidad vertiginosa \\
\hline la gran ola & dos olas gigantes \\
\hline ese furioso torrente & esa noche terrible \\
\hline
\end{tabular}

Como se ve en una masa café, furiosa, veloz, y en un 'pac, pac, pac' siniestro e imparable, puede haber dos o más Epítetos en un mismo grupo nominal. Asimismo, como ya adelantábamos, los Epítetos son graduables. En el ejemplo (8) se analiza un caso 
típico que involucra a un adverbio de grado, formando así un complejo hipotáctico de palabras $^{8}$ :

(8)

la mar muy linda
\begin{tabular}{|l|l|l|l|l|}
\hline grupo nominal & \multicolumn{2}{l|}{ clase de grupo } \\
\hline Deíctico & Cosa & \multicolumn{2}{|l}{ Epíteto } & función de grupo \\
\hline & & \multicolumn{2}{|c|}{ complejo de palabras } & clase compleja \\
\hline & & b & a & función compleja \\
\hline artículo & sust. común & adverbio & adjetivo & clase de palabra \\
\hline
\end{tabular}

Otros recursos de la estructura del grupo nominal tienen como función discursiva la de cuantificar o medir las entidades. La función primordial para estos efectos es el Numerativo. En español, el Numerativo precede a la Cosa (y a los Epítetos que la anteceden, si los hay). Esta función es realizada por la clase de los numerales, que involucran cuantificación $\mathrm{u}$ ordenación más precisa (segunda ola, 8 metros), o bien por un subconjunto de los cuantificadores indefinidos, que miden de manera más imprecisa y general (menos terror) ${ }^{9}$ :

Esta vez Ibarra alcanzó a "aproar" la nave y la pasaron con menos terror. Esta segunda ola tenía cerca de $\boldsymbol{8}$ metros de altura.

Cabe notar aquí dos contrastes importantes con la descripción de los Numerativos en inglés (Halliday y Matthiessen 2014). El primero dice relación con el hecho de que los Numerativos realizados por un mismo cuantificador pueden modificar tanto a sustantivos contables como de masa - p. ej. muchas calorías y mucha agua (en inglés 'many calories' y 'a lot of/much water', respectivamente). El segundo contraste atañe a la cuantificación comparativa. En el análisis del inglés, la comparación puede ser realizada por un complejo de palabras continuo (e.g. four more friends) o discontinuo (e.g. four friends more). En español, en cambio, el adverbio comparativo necesariamente debe aparecer postpuesto a la Cosa (p. ej. cuatro

\footnotetext{
${ }^{8}$ Por razones de espacio, no analizamos aquí otras posibilidades de realización de los Epítetos, que incluyen configuraciones estructurales de carácter apositivo (un día de mierda - un día muy de mierda) o focalizadas (el tonto de Juan), ni las variadas posibilidades de gradación de Epítetos adjetivales (morfológicas, léxicas y sintácticas) exploradas por Di Tullio (2012).

${ }^{9}$ Distinguimos aquí a este subconjunto de cuantificadores indefinidos que realiza la función Numerativo (menos, pocos, mucho, etc.) de otros cuantificadores indefinidos como alguno(s), uno(s), cualquier, todo(s), etc. que funcionan como determinantes indefinidos (Leonetti 2007). Desde un punto de vista sistémico-funcional, estos últimos permiten introducir entidades discursivas por medio de referencia inespecífica y, por tanto, realizan más bien a la función de Deíctico. Véase la sección 3.2 para el tratamiento general de esta función en relación con artículos, demostrativos y posesivos.
} 
amigos más). Para esta estructura, por tanto, proponemos en (9) el análisis de un Numerativo discontinuo, lo que evita postular una función comparativa cuantificadora separada, respetando así la relación de dependencia discontinua entre el numeral (cuatro) y el adverbio (más):

(9)

cuatro amigos más
\begin{tabular}{|l|l|l|l|}
\hline grupo nominal & ...ativo & función de grupo \\
\hline Numer... & Cosa & ...palabras & clase compleja \\
\hline complejo de... & & b & función compleja \\
\hline a & & adverbio & clase de palabra \\
\hline numeral & sust. común & &
\end{tabular}

\subsection{Recursos textuales del grupo nominal}

El reportaje que estamos empleando para ejemplificar los recursos del grupo nominal es un texto escrito que, como adelantamos en la presentación de esta sección, instancia una serie de géneros de la familia de los relatos (Martin y Rose 2008). En lo relativo a las entidades discursivas involucradas, esto significa que el texto en su totalidad debe, a grandes rasgos, proporcionar su propio contexto. El sistema semántico-discursivo en juego aquí es el sistema de IDENTIFICACIón (Martin 1992). Este sistema, como se muestra en la Figura 3, presenta la opción primaria entre la presentación de una entidad (cuya identidad no es recuperable por el(la) lector(a)/oyente en el discurso) o la recuperación de la entidad si su identidad es, por el contrario, recuperable:

Figura 3: Sistema de IDENTIFICACIón básico

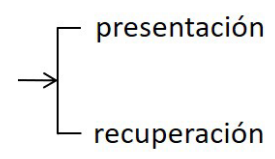

Los relatos de 'La ola maldita' usan sustantivos propios varias veces para presentar por primera vez a personas específicas. Solo una de ellas ya es identificable (exofóricamente) por la mayoría de los lectores chilenos - la Presidenta Bachelet - y solo dos de las personas que aparecen nombradas por medio de sustantivos propios ya habían sido introducidas en el discurso mediante referencia de presentación, como se ejemplifica en el extracto a continuación ${ }^{10}$ :

\footnotetext{
${ }^{10}$ La otra persona es Timmy, el hijo de Mariela Rojas. Se lo introduce en el texto por medio de uno (en uno de ellos, donde ellos recupera la referencia a ocho niños que juegan en la playa cuando Jonathan y sus amigos llegan a la isla Orrego), y luego es nombrado: De ellos no se sabe nada, salvo de uno: Timmy, de 4 años.
} 
Encontró ahi a una señora que con toda calma esperaba lo que el destino le ofreciera. Se llamaba Blanca... Blanca vivía sola y había aceptado morir sola.

Así, cuando no se emplean sustantivos propios para este propósito, la presentación de identidades en español requiere como mínimo la estructura Deíctico ${ }^{\wedge}$ Cosa. La función de Deíctico en la introducción de un referente nuevo requiere un determinante indefinido - en la terminología de Halliday y Matthiessen 2014, un determinante 'no específico': p. ej. el artículo indefinido una en (10). Una vez nombrada, esta entidad puede recuperarse por medio del nombre propio, como se analiza en (11):

una señora $[\ldots]$
\begin{tabular}{|l|l|l|l|}
\hline grupo nominal & clase de grupo \\
\hline Deíctico & Cosa & {$[\ldots]$} & función de grupo \\
\hline artículo & sust. común & & clase de palabra \\
\hline
\end{tabular}

(11)

Blanca
\begin{tabular}{|l|l|}
\hline grupo nominal & clase de grupo \\
\hline Cosa & función de grupo \\
\hline sust. propio & clase de palabra \\
\hline
\end{tabular}

La función Deíctico en español puede ser realizada por determinantes definidos o indefinidos ('específicos' o 'no específicos' en la terminología sistémico-funcional), lo que incluye artículos, cuantificadores, demostrativos y posesivos. Las posibilidades de realización por medio de determinantes definidos o indefinidos dependerán de si la entidad es recuperable (endofórica o exofóricamente) o bien si no lo es, p. ej. porque se introduce por primera vez (Martin 1992). La descripción sistémico-funcional del inglés también admite la realización del Deíctico por medio de grupos nominales incrustados (en las llamadas estructuras de 'genitivo', como 'The Pinita’s crew') pero en español la estructura equivalente es distinta y aquí, como ya vimos antes, la analizamos como Deíctico $\wedge$ Cosa ${ }^{\wedge}$ Cualificador, como en el grupo nominal los tripulantes del Pinita ${ }^{11}$.

\footnotetext{
${ }^{11}$ Parece que la función Cualificador, que en la descripción inglesa se aplica a todos los postmodificadores de la función Cosa, requiere una revisión más atenta en español, toda vez que en esta lengua hay distintos tipos de postmodificadores, como ya vimos en el caso de las funciones Clasificador y Epíteto (e incluso Numerativo o Deíctico). No nos detenemos aquí, por tanto, en la cuestión de si es necesario o no distinguir una función diferente del Cualificador para otros tipos de frases preposicionales con $d e$, p. ej. aquellas que involucran relaciones de 'posesión' (entendida en sentido amplio, es decir, incluidas las relaciones de 'todo/parte' y de 'contenedor/contenido'). Véase Fontaine (2017) para una discusión análoga sobre las posibilidades descriptivas para los postmodificadores en el grupo nominal inglés.
} 
En 'La ola maldita', muchas veces se presentan también personas por primera vez en relación con otras entidades que ya han sido presentadas antes, por medio de un posesivo + una estructura apositiva: su capitán José Ibarra; su hijo Jonathan Romero; su nieto de 4 años, Emilito; su madre, Mariela Rojas. Cuando lo anterior no es posible, se despliegan estructuras apositivas en las que es la función Cosa (incluidos algunos postmodificadores) la que permite clarificar información para identificar a la persona en cuestión, como en el pescador Mario Quiroz Leal; el contralmirante Roberto Macchiavello; el piloto Victor González; el intendente de Concepción, Jaime Tohá; el sargento de la Armada, Cristián Valladares. Estas estrategias son más eficientes que la estrategia que involucra primero presentar y después nombrar (como en el ejemplo con Blanca, más arriba). También evita tener que depender solo de un nombre propio, lo que involucraría decirle al lector que debe recuperar la identidad de alguien que en realidad le es desconocida.

Una vez nombrada, puede emplearse, desde luego, el nombre propio para recuperar y mantener la referencia de la persona en cuestión. El capitán del bote pesquero que queda atrapado en medio del tsunami, de hecho, se va recuperando de esta manera en la siguiente secuencia dentro del reportaje: El bote pesquero Pinita - su capitán José Ibarra - el capitán Ibarra - Ibarra - Ibarra - Ibarra - Ibarra - Ibarra - el capitán Ibarra - el capitán IbarraIbarra - Ibarra.

Ibarra
\begin{tabular}{|l|l|}
\hline grupo nominal & clase de grupo \\
\hline Cosa & función de grupo \\
\hline sust. propio & clase de palabra \\
\hline
\end{tabular}

Como ya hemos visto, la gramática interna de los nombres propios en español incluye regularmente un determinante definido cuando se especifica el rol de la persona que se está identificando: la Presidenta Bachelet, el sargento Valladares. Este patrón plantea también una diferencia con lo que ocurre con en inglés con grupos nominales que incluyen nombres propios de manera análoga, ya que en esta lengua no admiten determinantes: *the president Bachelet, ${ }^{*}$ the sergeant Valladares (Halliday y Matthiessen 2014).

Las entidades discursivas pueden recuperarse discursivamente en español por medio de pronombres personales (llamados 'tónicos') que, a diferencia de los pronombres clíticos, pueden realizar la función Cosa dentro de la estructura del grupo nominal:

...recuerda Hugo Barrera, un sobreviviente... Hugo cayó a ese furioso torrente sabiendo que moriría. Él estaba en Orrego por trabajo. 
(13)

$e ́ l$
\begin{tabular}{|l|l|}
\hline grupo nominal & clase de grupo \\
\hline Cosa & función de grupo \\
\hline pronombre & clase de palabra \\
\hline
\end{tabular}

Además de los sustantivos propios y los pronombres personales, el español despliega una amplia gama de recursos para recuperar la identidad de las entidades discursivas. En el extracto que se presenta a continuación, el anciano rescatado por el sargento Valladares es presentado primero por medio de un artículo indefinido (un anciano [...]) y luego recuperado por medio de dos determinantes posesivos (sus familiares, sus parientes), cuatro clíticos pronominales, lo, lo, lo, lo (lo cuidaba, lo encontró, lo sacó, lo llevó), un artículo y un demostrativo: el, ese (al anciano, ese anciano):

En su retirada, el sargento Valladares vio que una mujer pedia ayuda. Al entrar en la casa medio derrumbada, encontró a un anciano en silla de ruedas que los miraba con angustia. Sus familiares estaban en los cerros. La señora que pedia auxilio era la mujer que lo cuidaba. Ella vivía en otro lugar y corrió a ver al anciano y lo encontró solo. Estaba mojado y gemía de miedo. El sargento lo sacó de ahi y lo llevó a la casa de la mujer.

\section{El saqueo}

No es dificil imaginar la angustia de ese anciano abandonado. Tampoco es incomprensible el miedo que deben haber sentido sus parientes.

A la mujer que cuida al anciano se la presenta y recupera en una secuencia similar, que incluye el pronombre personal ella: una mujer - los - la señora que pedía auxilio - la mujer que lo cuidaba - ella - la mujer. Analizamos el grupo nominal correspondiente en (14):

ella
\begin{tabular}{|l|l|}
\hline grupo nominal & clase de grupo \\
\hline Cosa & función de grupo \\
\hline pronombre & clase de palabra \\
\hline
\end{tabular}

Es posible establecer dos contrastes importantes con las descripciones sistémicofuncionales del grupo nominal inglés: en primer lugar, en español, las entidades pueden ser recuperadas en el discurso por medio de pronombres personales al interior de grupos nominales, pero es muy común que sean recuperadas por medio de clíticos pronominales, 
dentro de la estructura del grupo verbal (Quiroz 2017b). Los pronombres personales ('tónicos') son mucho menos usados en español que en inglés y, en nuestra lengua, se limitan primordialmente a entidades que corresponden a personas (o a entidades 'personificadas') ${ }^{12}$, mientras que los clíticos pronominales no presentan esta limitación. Un segundo contraste dice relación con la recuperabilidad de entidades realizadas por distinciones de persona en la flexión verbal (cuando el grupo verbal es finito). Este recurso puede emplearse para recuperar entidades es dentro y entre complejos de cláusulas. Las identidades de la mujer y del sargento son recuperadas de esta forma en (16) y (17) a continuación (las entidades recuperadas aparecen subrayadas y la flexión que las recupera en negrita):

(16) Ella vivía en otro lugar y corrió a ver al anciano y lo encontró solo.

(17) El sargento lo sacó de ahi y lo llevó a la casa de la mujer.

En (18), tanto la identidad del sargento como la del anciano son recuperadas entre complejos de cláusulas, es decir, claramente por sobre los límites oracionales:

(18) Al entrar en la casa medio derrumbada, encontró a un anciano en silla de ruedas que los miraba con angustia. Estaba mojado y gemía de miedo.

Este tipo de recuperación de entidades entre complejos de cláusulas sería agramatical en la lengua inglesa, que requiere una función Sujeto en estructuras comparables (véase descripción del modo en inglés, p. ej. Halliday y Matthiessen 2014). La estructura interpersonal de la cláusula española, en cambio, no requiere dicha función para realizar opciones de MODO ni para negociar la responsabilidad modal en el intercambio dialógico (Quiroz 2015, 2017a). Y dado que no hay 'ausencia' de algo, sino más bien la presencia de distinciones morfológicas claras, no se justifica el análisis de una entidad 'elidida' (como suele hacerse en los manuales de lingüística textual del español, que basan el análisis de la elipsis en los patrones traducidos del trabajo llevado a cabo en inglés, incluido el de Halliday y Hasan 1976).

El cuadro 2 sintetiza los principales patrones en español para presentar y recuperar referentes ejemplificados por medio de las entidades del anciano y su cuidadora en el texto 'La ola maldita':

\footnotetext{
${ }^{12}$ El pronombre neutro de 3ra persona ello se emplea en registros muy acotados y se restringe a la referencia textual (Martin 1992); no aplica a la referencia a entidades.
} 
Cuadro 2. Dos instancias de recuperación de entidades en español

\begin{tabular}{|c|c|c|c|c|}
\hline \multirow[t]{2}{*}{ Presentación } & \multicolumn{4}{|l|}{ recuperación } \\
\hline & determinantes & $\begin{array}{l}\text { pronombres } \\
\text { personales }\end{array}$ & $\begin{array}{l}\text { clíticos } \\
\text { pronominales }\end{array}$ & $\begin{array}{l}\text { flexión } \\
\text { verbal }\end{array}$ \\
\hline \multicolumn{5}{|l|}{ una mujer } \\
\hline & & & los & \\
\hline & la (señora)... & & & \\
\hline & $=l a($ mujer $) \ldots$ & & & \\
\hline & & ella & & \\
\hline & & & & $-i o ́$ \\
\hline & & & & $-o ́$ \\
\hline & la (mujer) & & & \\
\hline \multicolumn{5}{|l|}{ un anciano } \\
\hline & sus (familiares) & & & \\
\hline & & & lo & \\
\hline & al (anciano) & & & \\
\hline & & & lo & \\
\hline & & & & $\begin{array}{l}-a b a \\
-i a\end{array}$ \\
\hline & & & lo & \\
\hline & & & lo & \\
\hline & ese (anciano)... & & & \\
\hline & sus (parientes) & & & \\
\hline
\end{tabular}

En la subsección anterior ya adelantábamos que el grupo nominal español puede desplegar la función Cualificador cuando se requiere información adicional que especifique qué entidad está siendo recuperada. En el reportaje de Guzmán (2010), este recurso es útil cuando el sargento Valladares se da cuenta de que la mujer que ve pidiendo ayuda es la cuidadora del anciano que está rescatando: la señora que pedía auxilio. El Cualificador que pedía auxilio especifica referencialmente qué mujer, ya que en el resto del reportaje hay varias candidatas posibles:

En su retirada, el sargento Valladares vio que una mujer pedia ayuda. Al entrar en la casa medio derrumbada, encontró a un anciano en silla de ruedas que los miraba con 
angustia. Sus familiares estaban en los cerros. La señora que pedía auxilio era la mujer que lo cuidaba. Ella vivía en otro lugar y corrió a ver al anciano y lo encontró solo.

El ejemplo destacado en el extracto es comparable a un anciano en silla de ruedas que los miraba con angustia, presentado más arriba, salvo que, en este segundo caso el grupo nominal presenta una entidad por primera vez. Por lo tanto, sus Cualificadores en silla de ruedas (frase preposicional) y que los miraba con angustia (cláusula de relativo) acotan ideacionalmente la identidad de la entidad, no fóricamente.

En las descripciones sistémico-funcionales, la función de Cualificador es, lexicogramaticalmente, un postmodificador realizado por unidades incrustadas, es decir, por unidades desplazadas de rango. Se habla de desplazamiento de rango (rank-shift) porque las unidades en cuestión pueden funcionar dentro de la estructura de una unidad menor como la cláusula de relativo restrictiva que pedía auxilio, analizada en (15):

(15)

la señora
\begin{tabular}{|l|l|l|l|}
\hline \multicolumn{2}{|l|}{ grupo nominal pedía auxilio } \\
\hline Deíctico & Cosa & Cualificador & clase de grupo \\
\hline artículo & sust. común de grupo & {$[$ [cláusula] $]$} & clase de palabra \\
\hline
\end{tabular}

En (15), se emplean convencionalmente corchetes dobles para indicar que la unidad incrustada que realiza la función de grupo es una cláusula. La unidad incrustada como Cualificador también puede corresponder a una frase preposicional, desplazada para funcionar en la estructura de una unidad de su mismo rango (grupo/frase). En (16), la frase preposicional del Pinita ejemplifica esta opción, con corchetes simples para indicar que se trata de una unidad del rango de grupo/frase:

los tripulantes
\begin{tabular}{|l|l|l|l|}
\hline grupo nominal & del Pinita \\
\hline Deíctico & Cosa & Cualificador & función de grupo \\
\hline artículo & sust. común & [frase prep.] & clase de palabra \\
\hline
\end{tabular}

La frase preposicional incrustada en (16) proporciona, discursivamente, un apoyo comparable a la cláusula de relativo de (15), por cuanto clarifica quiénes o cuáles son los tripulantes recuperados en el grupo nominal los tripulantes del Pinita. En otras palabras, restringe su referencia de un modo más preciso que lo que podría hacer la función Deíctico por sí sola. 
Hay un último patrón que aquí podemos contrastar con la descripción sistémicofuncional del grupo nominal inglés. El español posee un paradigma de determinantes posesivos ciertamente similar, conocido tradicionalmente como el de los adjetivos posesivos', p. ej. mi familia, tu mujer, su ciudad, sus vidas, nuestro grupo, nuestras costas. Como seńalan Halliday y Hasan (1976), los posesivos siempre remiten a dos referentes, porque hacen referencia tanto al 'poseedor' como a lo 'poseído'. Así, desde un punto de vista semántico-discursivo, estos determinantes posesivos recuperan la identidad de dos entidades: la realizada por medio de la función Cosa (familia) y otra realizada por medio de la función Deíctico:

familia
\begin{tabular}{|l|l|l|}
\hline \multicolumn{2}{|l|}{ grupo nominal } & clase de grupo \\
\hline Deíctico & Cosa & función de grupo \\
\hline posesivo & sust. común & clase de palabra \\
\hline
\end{tabular}

Las clases de posesivos dentro de este paradigma distinguen persona y, como vimos en los ejemplos, realizan la función Deíctico antepuesta a la función Cosa. Pero hay un segundo paradigma de posesivos en español, la de los posesivos 'tónicos', que puede funcionar como núcleo del grupo nominal - p. ej. en la cláusula el bote pesquero es suyo ${ }^{13}$, o pueden aparecer pospuesto a la Cosa, como en el bote pesquero suyo. En el análisis no sistémico, que no distingue entre clase y función en una escala de rango, se trata a los primeros como 'pronombres' posesivos y a los segundos como 'adjetivos (determinativos)'. Estos posesivos además de distinguir número, distinguen género en todas las personas: mio(s), mia(s), tuyo(s), tuya(s), suyo(s), suya(s), nuestro(s), nuestra(s).

Cuando estos posesivos acentuados aparecen postpuestos a la función Cosa, pueden especificar referencialmente aún más al elemento que ya va especificado mediante un Deíctico en posición inicial, como se adelantaba en el ejemplo el bote pesquero suyo, más arriba, analizado con mayor detalle en (18). Desde el punto de vista semántico-discursivo, se recuperan de nuevo dos entidades, una mediante la función Deíctico + la función Cosa, y otra por medio del posesivo suyo:

\footnotetext{
${ }^{13}$ No ahondamos aquí en la cuestión de la diferenciación entre los posesivos tónicos que pertenecen a la clase de los 'pronombres' y a los que pertenecen a la clase de los 'adjetivos' (o 'adjetivos determinativos'), respectivamente, ya que ello implica abordar con mayor detención la distinción entre grupos nominales elípticos y no elípticos. Dirigimos al lector/la lectora a la revisión de los posesivos en español que presentan Picallo y Rigau (1999), quienes categorizan las distintas clases de posesivos desde una perspectiva más bien formal.
} 
(18)

\begin{tabular}{|c|c|c|c|c|}
\hline el & bote & pesquero & suyo & \\
\hline \multicolumn{4}{|c|}{ grupo nominal } & clase de grupo \\
\hline Deíctico & Cosa & Clasificador & Deíctico & función de grupo \\
\hline artículo & sust. común & adjetivo & posesivo & clase de palabra \\
\hline
\end{tabular}

El primer Deíctico en la estructura también puede ser realizado por un demostrativo, como en (19):

ese bote pesquero
\begin{tabular}{|l|l|l|l|l|}
\hline grupo nominal & suyo & clase de grupo \\
\hline Deíctico & Cosa & Clasificador & Deíctico & función de grupo \\
\hline demostrativo & sust. común & adjetivo & posesivo & clase de palabra \\
\hline
\end{tabular}

Lo anterior plantea la cuestión de cómo analizar esta función postpuesta a la Cosa cuando ya hay una función Deíctico antepuesta (mediante artículo definido o demostrativo). Cabe notar que, a diferencia del Numerativo cuatro.. más en (9), aquí no parece que tengamos un constituyente funcional realizado de manera discontinua. Más bien se da una especie de especificación adicional de la deixis, donde el artículo definido el en (18) o el demostrativo ese en (19) se emplean para identificar referencialmente a la entidad bote pesquero en posición inicial, mientras que el posesivo suyo se usa para identificar a dicha entidad por medio de relaciones de posesión más específicas, porque incorpora a una segunda entidad. Es interesante que, a diferencia del inglés, no se requiera en español un indicador de función para relacionar al poseedor con la Cosa (cf. of his), aunque sí es, desde luego, posible en estructuras más claramente contrastivas: el bote de él (no de otro). Por tanto, proponemos aquí que estas estructuras involucran deixis secundaria a través de una segunda función Deíctico situada hacia el final del grupo nominal. Esta especie de 'doble' deixis también puede involucrar a determinativos demostrativos. Compárese ese bote pesquero con el bote pesquero ese, analizado en (20):

\begin{tabular}{|c|c|c|c|c|}
\hline el & bote & pesquero & ese & \\
\hline \multicolumn{4}{|c|}{ grupo nominal } & \multirow{2}{*}{$\begin{array}{l}\text { clase de grupo } \\
\text { función de grupo }\end{array}$} \\
\hline Deíctico & Cosa & Clasificador & Deíctico & \\
\hline artículo & sust. común & adjetivo & demostrativo & clase de palabra \\
\hline
\end{tabular}


Como se observa en (18)-(20), si se despliegan dos Deícticos dentro de la estructura del grupo nominal español, el segundo es necesariamente más específico que el primero en la secuencia. Compárese ese...suyo y el...ese. Estas posibilidades sugieren una escala de menor a mayor especificidad referencial: 'artículo definido' < 'demostrativo' < 'posesivo'.

Hemos revisado hasta aquí el lugar que tienen funciones claves del grupo nominal para recuperar la identidad de entidades en el discurso, primordialmente mediante opciones básicas en el sistema semántico-discursivo de idENTIFICACión. Estas funciones incluyen a la Cosa (cuando es realizada por un pronombre personal 'tónico' o un sustantivo propio), al Deíctico y al Cualificador. La función de Deíctico en particular involucra primordialmente a la clase de los determinantes (Leonetti 1999): artículos, demostrativos y posesivos, que en espańol requieren seleccionar un mínimo de información (solo persona, número y, opcionalmente, género) para identificar la entidad discursiva que están recuperando. En cuanto a la función de Cualificador, que fue introducida aquí en relación con opciones en IDENTIFICACIÓN (metafunción textual), podría plantearse que su presencia en la estructura también puede estar motivada por opciones de semántico-discursivas en IDEACión (metafunción ideacional). Así, cuando la función de Cualificador aparece en grupos nominales que incluyen a un Deíctico realizado por un determinante definido, acota la identidad de una entidad en términos primordialmente textuales, p. ej. en el grupo nominal la señora que pedía auxilio, que recupera fóricamente la identidad de una entidad desde el discurso previo dentro del reportaje. En cambio, si el Deíctico es realizado por un determinante indefinido, puede plantearse que el Cualificador acota la identidad de la entidad clarificando información de índole ideacional ('léxico'), p. ej. el grupo nominal un anciano en silla de ruedas que los miraba con angustia, que en el reportaje introduce una entidad por primera vez y cuyos Cualificadores la especifican ideacionalmente.

\section{Sistemas DEL GRUPO NOMINAL}

Siguiendo la perspectiva 'trinocular' adoptada en las descripciones sistémicofuncionales, en las secciones anteriores hemos abordado la estructura del grupo nominal 'por arriba', 'por alrededor' y 'por debajo' (Halliday 1992, 1996; Caffarel et al. 2004). La perspectiva 'por arriba' partió desde los distintos recursos funcionales que conforman potencialmente su estructura y que contribuyen de maneras específicas a la realización de opciones en sistemas semántico-discursivos de IDEACIÓN e IDENTIFICACIÓN. El abordaje del grupo nominal 'por alrededor' y 'por debajo' implicó acercarse tanto desde el punto de vista de las funciones estructurales básicas que lo conforman (y las relaciones entre dichas funciones), como desde el punto de vista de las clases de unidades que las realizan a lo largo de la escala de rango del español. En síntesis, el potencial funcional de la estructura del grupo nominal en español puede generalizarse de la siguiente manera:

$$
\text { Deíctico }(\wedge \text { Epíteto }) \wedge \text { Cosa } \wedge \text { Clasificador } \wedge \text { Epíteto }(\wedge \text { Deíctico }) \wedge \text { Cualificador }
$$


Hasta aquí, los sistemas de opciones subyacentes han quedado relativamente implícitos, de manera que en esta última sección el foco estará en explicitar la organización paradigmática que subyace a la estructura del grupo nominal, tal y como la hemos explorado aquí.

En primer lugar, en la descripción sistémica que proponemos en la Figura $4^{14}$ dejaremos fuera a los grupos nominales elípticos, que no hemos abordado aquí. Siguiendo a Halliday y Hasan (1976), entendemos por grupos nominales elípticos a aquellos en los que la función Cosa es recuperada por medio de su omisión. En este sentido, hemos dejado fuera de la revisión propuesta aquí, por razones de espacio, a todos los grupos nominales en los que podríamos considerar que se da este tipo de elipsis y que tienen, por tanto, a otras funciones por núcleo: Deíctico (el Æ suyo, ese Æ), Epíteto (la Æ roja, los Æ grandes), Numerativo (los dos Æ, muchos Æ) y Cualificador (los Æ de otras cuatro personas) ${ }^{15}$. Dentro de los no elípticos, distinguimos entre grupos nominales designativos y especificativos, es decir, entre aquellos que designan a una entidad recuperable y los que especifican a una entidad. Los grupos nominales designativos, a su vez, se dividen en aquellos que emplean un sustantivo propio para denominar a una entidad y los que emplean un pronombre personal:

Figura 4. Sistemas generales del grupo nominal en español

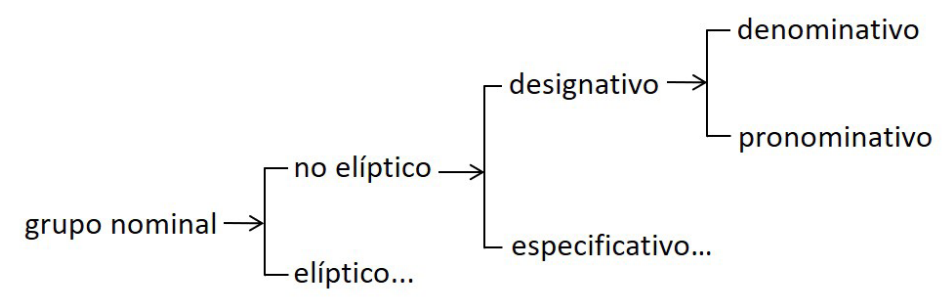

El cuadro 3 a continuación presenta los enunciados de realización que vinculan las opciones asociadas en este punto al rasgo sistémico [no elíptico] con sus consecuencias estructurales:

\footnotetext{
${ }^{14}$ Aquí seguimos a Quiroz (2016) en las convenciones sistémico-funcionales empleadas en español, tanto para las redes sistémicas de las Figuras 4 y 5, como para los enunciados de realización de los cuadros 3 y 4.

${ }^{15}$ Dejamos también fuera de la revisión (y discusión) la cuestión de tratar como elípticos o no, y en qué condiciones, a los grupos nominales que tienen como núcleo a una función distinta de la Cosa en ciertos entornos específicos, p. ej. en los Atributos adjetivales de cláusulas relacionales atributivas: El bote pesquero es suyo, Era fea la ola. Entendemos que la mirada que proponemos en este artículo, que privilegia una perspectiva discursiva a estos recursos, podría iluminar el debate sobre la elipsis en el ámbito del grupo nominal en español.
} 
Cuadro 3. Enunciados de realización para el rasgo [no elíptico: designativo] en el grupo nominal espańol

\begin{tabular}{|l|l|}
\hline no elíptico & + Cosa \\
\hline denominativo & Cosa: sustantivo propio \\
\hline pronominativo & Cosa: pronombre personal \\
\hline
\end{tabular}

El rasgo [especificativo] abre opciones en cuatro sistemas más delicados. Esto permite, por medio de distintas configuraciones estructurales del grupo nominal, que las entidades discursivas sean clasificadas, descritas, cuantificadas, determinadas y(o) cualificadas. Como ya hemos adelantado en la sección 3.1, los rasgos [clasificado] y [descrito] corresponden en realidad a opciones recursivas, ya que puede requerirse más de un Clasificador o Epíteto (no formalizamos este carácter recursivo en la red sistémica de la Figura 5). El rasgo [clasificado] puede especificarse aún más en la opción entre [adjetival] o [nominal], para admitir Clasificadores realizados por adjetivos (generador eléctrico) o por sustantivos (silla de ruedas, coche bomba). El rasgo [descrito] se subclasifica provisionalmente en [descriptivo] o [actitudinal], para reflejar las posibles implicaciones de esta distinción en la interpretación de ciertos adjetivos cuando realizan Epítetos antepuestos (p. ej. un pobre amigo vs un amigo pobre). El rasgo [cuantificado] se subclasifica en [exacto] o [inexacto] para distinguir la cuantificación u ordenación precisa (p. ej. la segunda ola, 8 metros), de la general (menos terror). Admitimos la posibilidad de un sistema comparativo opcional para dar cuenta de los Numerativos discontinuos (cuatro amigos más). El rasgo [determinado] se subclasifica en [personal] o [general] para distinguir entre los grupos nominales que realizan dos entidades (p. ej. sus familiares) de los que realizan una sola (ese bote pesquero). Un sistema más delicado dependiente del rasgo [+determinado] abre el espacio a grupos nominales con deixis secundaria, ya sea posesiva o demostrativa, postpuesta a la función Cosa. El rasgo [cualificado] se especifica en [frasal] o [clausal]. La Figura 5 organiza todos estos sistemas más específicos y sus interdependencias: 
Figura 5. Sistemas del grupo nominal más delicados para el español.

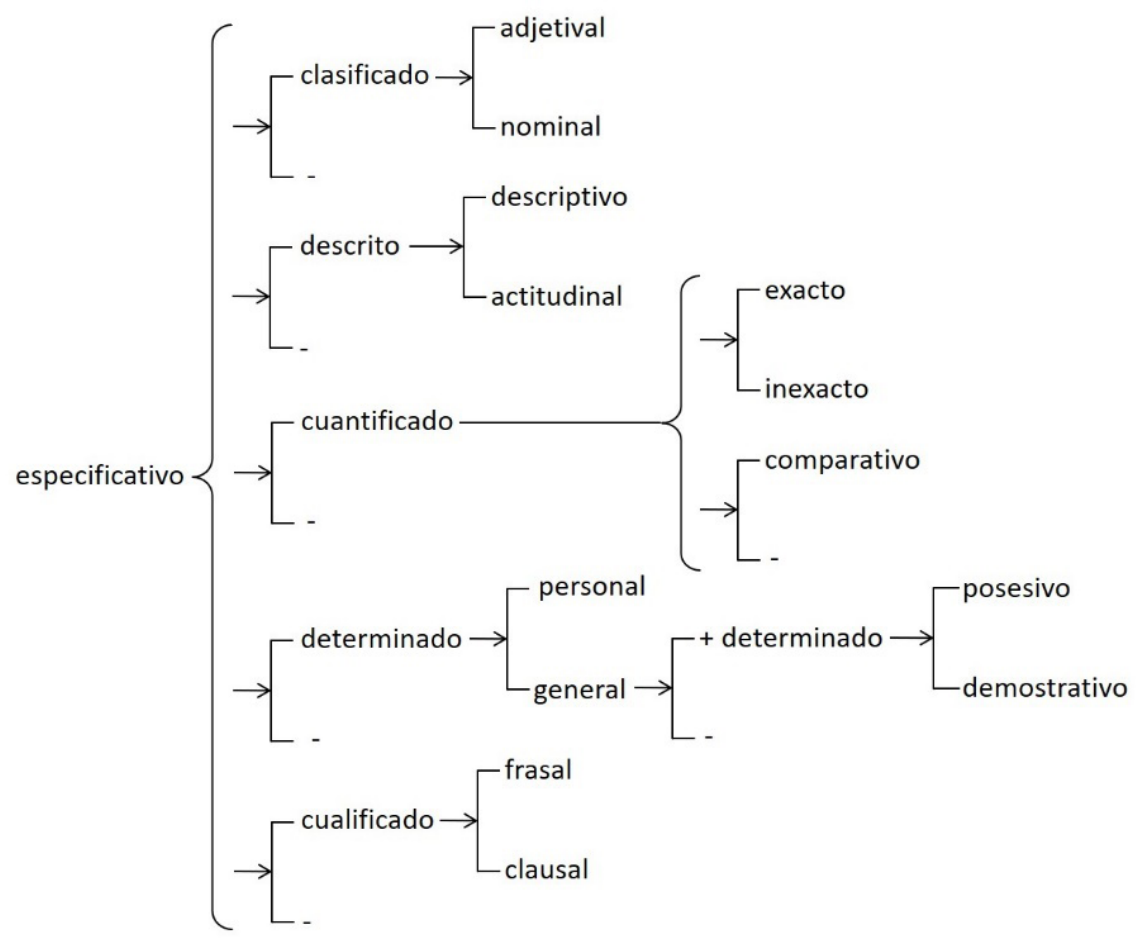

Los enunciados de realización que vinculan a cada uno de estos rasgos sistémicos con sus consecuencias estructurales se presentan en el cuadro 4. El símbolo '\#' indica el inicio o final del grupo nominal, ' + ' indica la presencia de una función, ' $\wedge$ ' indica la secuencia relativa que siguen las funciones, ':' especifica la realización de una función por medio de una clase en un rango inferior. Aquí empleamos también las flechas ${ }^{(`)} \mathrm{o}$ ‘ $\urcorner$ ' para representar relaciones de dependencia entre funciones en la secuencia, de modo que ' $\mathrm{X}$ ' $\mathrm{Y}$ ' significa ' $\mathrm{X}$ depende de Y', y viceversa:

Cuadro 4. Enunciados de realización para los rasgos más delicados del grupo nominal

\begin{tabular}{|l|l|}
\hline clasificado & + Clasificador; Cosa $\wedge$ Clasificador \\
\hline adjetival & Clasificador: adjetivo \\
\hline nominal & Clasificador: $d e^{\wedge}$ sustantivo \\
\hline descrito & + Epíteto; Epíteto ${ }^{\wedge}$ Cosa $o$ bien Cosa $\urcorner$ Epíteto \\
\hline
\end{tabular}




\begin{tabular}{|c|c|}
\hline descriptivo & Epíteto: adjetivo calificativo \\
\hline actitudinal & Epíteto: adjetivo calificativo evaluativo; adjetivo adverbial \\
\hline cuantificado & +Numerativo; Numerativo Epíteto \\
\hline exacto & Numerativo: numeral \\
\hline inexacto & Numerativo: cuantificador \\
\hline comparativo & Numerativo: complejo de palabras; Numer... Cosa $\urcorner$...ativo \\
\hline determinado & +Deíctico; Deíctico ${ }^{\circ}$ Numerativo \\
\hline personal & Deíctico: determinante: posesivo \\
\hline general & Deíctico: determinante: no posesivo \\
\hline + determinado & +Deíctico; Deíctico $\wedge$ \# \\
\hline posesivo & Deíctico: posesivo tónico \\
\hline demostrativo & Deíctico: demostrativo \\
\hline cualificado & +Cualificador: Cosa $\urcorner$ Cualificador \\
\hline frasal & Cualificador: [frase preposicional] \\
\hline clausal & Cualificador: [[clausal]] \\
\hline
\end{tabular}

\section{Conclusiones}

El objetivo de este trabajo fue ofrecer un perfil integrado del grupo nominal en español que tuviera en cuenta la interacción entre principios teóricos claves de la LSF. Desde la perspectiva de las metafunciones ideacional y textual (dimensión de metafunción), se consideró la interacción de los correspondientes sistemas de IDEACIÓN e IDENTIFICACIÓN en el estrato semántico-discursivo con la organización interna del grupo nominal en el estrato lexicogramatical (dimensión de estratificación). La organización interna del grupo nominal, a su vez, fue descrita tanto desde el punto de vista de su estructura - la configuración orgánica de funciones realizadas por diversas clases de unidades en rangos inferiores (dimensión de escala de rango) - como desde el punto de vista sistémico - es decir, de la organización paradigmática subyacente a las posibilidades estructurales examinadas (dimensión de eje). Todo lo anterior se ilustró mediante los recursos instanciados en un reportaje conformado por diversos géneros de la familia de los relatos en español de Chile (dimensión de instanciación).

La perspectiva discursiva tiene dos grandes ventajas. La primera de ellas ya ha sido señalada por Martin (1983) en cuanto a la importancia de tomar en serio, en la descripción de la lexicogramática de distintas lenguas, los patrones discursivos como punto de partida, particularmente en el trabajo de orientación funcional con vocación tipológica. Esto porque, si bien las funciones discursivas básicas son compartidas por todos los(las) hablantes/ escritores(as) al momento de producir textos situados, los recursos lexicogramaticales 
específicos movilizados al servicio de dichas funciones varían notablemente de una lengua a otra. Partir desde patrones discursivos previene de manera mucho más eficaz la imposición de categorías gramaticales - que han evolucionado a partir del estudio de una lengua (o grupo de lenguas) - a la descripción de una lengua diferente. Retomando así el legado de Whorf (1945), lo que es 'común' o generalizable entre distintas lenguas tiene así una base empíricamente más sólida: no fundamentada en nociones 'universales' interpretables, sino en patrones observados en textos situados.

Todo lo anterior lleva a la segunda ventaja: la posibilidad de evaluar de manera más sistemática la convergencia y la divergencia dentro del mismo estrato lexicogramatical. Por una parte, las funciones estructurales descritas para el grupo nominal español, así como las opciones básicas en los sistemas subyacentes son, en efecto, comparables en más de un sentido. Hemos intentado mostrar aquí que funciones estructurales básicas del grupo nominal - Cosa, Clasificador, Epíteto, Numerativo, Deíctico y Clasificador -, e incluso las opciones más básicas en el sistema que las motiva, son comparables con las del grupo nominal descrito en inglés. Sin embargo, la descripción que aquí presentamos del grupo nominal español también guarda diferencias importantes con la del inglés en cuanto a i) la relación que establecen estas funciones en la estructura (incluida su secuenciación relativa) y ii) las clases de unidades y los sintagmas que pueden realizar estas funciones a lo largo de la escala de rango. A este respecto, la flexibilidad que ofrece la distinción sistémico-funcional entre funciones estructurales y clases de unidades que realizan dichas funciones en la escala de rango (Martin 2004) nos ha permitido mostrar con mayor claridad en qué sentido el grupo nominal español difiere del grupo nominal en inglés (y, potencialmente, en otras lenguas).

Hemos privilegiado, en este primer acercamiento, un enfoque más bien generalista u 'holístico' (Halliday 2009), lo que ha implicado, necesariamente, dejar fuera muchos recursos y patrones más detallados del grupo nominal español que podrían incluso sugerir funciones o configuraciones estructurales no descritas con anterioridad, simplemente porque no se dan en el grupo nominal inglés (Halliday y Matthiessen 2014). Lo anterior va unido a la necesidad de basar la descripción de los grupos nominales en registros y(o) géneros diversos, de manera de poder desarrollar una descripción mucho más detallada y explícita que pueda emplearse en el análisis de textos diferentes, sobre todo en investigaciones aplicadas. Aquí hemos optado por sustentar nuestra descripción sobre la base de ejemplos tomados de un texto en particular, sin siquiera abordar los patrones asociados a todos los grupos nominales que allí aparecen. Tenemos plena conciencia que este es solo el punto de partida ante el desafío que se abre por delante y que implica ir profundizando el estudio de grupos nominales más diversos y complejos en español.

\section{Obras citadas}

Bartoš, Lubomír. 1980. "La atribución relacional y el adjetivo de relación en el español”. Études Romanes de Brno 11: 69-77. 
Bosque, Ignacio. 1993. "Sobre las diferencias entre los adjetivos relacionales y los calificativos”. Revista Argentina de Lingüistica 9: 9-48.

Caffarel, Alice, J. R. Martin y C. M. I. M. Matthiessen. 2004. "Introduction: systemic functional typology”. En Alice Caffarel, J. R. Martin y C. M. I. M. Matthiessen, Eds., Language typology: a functional perspective. Ámsterdam: John Benjamins. 1-76.

Demonte, Violeta. 1999. "El adjetivo: clases y usos". En Ignacio Bosque y Violeta Demonte, Eds., Gramática descriptiva de la lengua española, Vol. 1. Madrid: Espasa-Calpe. 129-215

Di Tullio, Ángela. 2012. "Orden y concordancia en secuencias de adjetivos". En Mar Campos Souto, Ramón Mariño Paz, José Ignacio Pérez Pascual y Antonio Rifón Sánchez, Eds., Assi como es de suso dicho: estudios de morfología y léxico en homenaje a Jesús Pena. La Rioja: Cilengua. 179-193.

Doran, Yaegan y J. R. Martin. 2021. "Field relations: understanding scientific explanations”. En Karl Maton, J. R. Martin y Yaegan Doran, Eds., Teaching Science: language, knowledge, pedagogy. Londres: Routledge. 105-133.

Figueredo, Giacomo, Cristiano Araújo y Adriana Pagano. 2008. "Principles underlying the experiential and the logical structures of the nominal group in Portuguese". Proceeedings of the 19th European Systemic Functional Linguistics Conference and Workshop. Saarbrücken: Universität des Saarlandes. En línea: <https://publikationen.sulb.unisaarland.de/handle/20.500.11880/26765>

Fontaine, L. 2017. “The English nominal group: the centrality of the Thing element”. En Tom Bartlett y Gerard O'Grady, Eds., The Routledge Handbook of Systemic Functional Linguistics. Londres: Routledge. 267-283.

Guzmán, Juan Andrés. 2010. "La ola maldita". Paula. 19 de noviembre de 2019. <https:// www.latercera.com/paula/la-ola-maldita/>

Halliday, M. A. K. 1989. Spoken and written language. Segunda. Oxford: Oxford University Press.

. 1992. "Systemic grammar and the concept of a 'science of language". En Jonathan Webster, 2003, Ed., On language and linguistics Londres: Continuum. 199-212. . 1996. "On grammar and grammatics". En Jonathan Webster, 2002, Ed., On grammar. London: Continuum pp. 384-417.

. 1998. "Things and relations: regrammaticising experience as technical knowledge”. En J. R. Martin y Robert Veel, Eds., Reading science: critical and functional perspectives on discourses of science. Londres: Routledge. 185-235

. 2009. "Methods - techniques - problems". En M. A. K. Halliday y Jonathan Webster, Eds., Continuum companion to systemic functional linguistics. Londres: Continuum, 59-86.

Halliday, M. A. K. y Ruqaiya Hasan. 1976. Cohesion in English. Londres: Longman.

Halliday, M. A. K. y C. M. I. M. Matthiessen. 2014. Halliday's introduction to functional grammar. 4ta ed. Londres: Routledge.

Hao, Jing. 2020. Analysing scientific discourse from a Systemic Functional Linguistic perspective: A framework for exploring knowledge building in Biology. Londres: Routledge. 
Hernanz, María Lluïsa y José María Brucart. 1987. "El sintagma nominal”. En La sintaxis, Vol. I. Barcelona: Crítica. 142-209.

Lavid, Julia, Jorge Arús y Juan Rafael Zamorano Mansilla. 2010. Systemic functional description of Spanish: a contrastive study with English. Londres: Continuum.

Leonetti, Manuel. 1999. Los determinantes. Madrid: Arco Libros. . 2007. Los cuantificadores. Madrid: Arco Libros.

Martin, J. R. 1983. "Participant identification in English, Tagalog and Kâte". Australian Journal of Linguistics, 3.1: 45-74. doi: https://doi-org.ezproxy.puc. $\mathrm{cl} / 10.1080 / 07268608308599299$ . 1992. English text: system and structure. Ámsterdam: John Benjamins.

Martin, J. R. 1993a. "Life as a noun: arresting the universe in science and humanities". En M. A. K. Halliday y J. R. Martin, Eds., Writing science: literacy as discursive power. Londres: Taylor and Francis. 242-293.

1993b. "Technicality and abstraction: language for the creation of specialized texts". En M. A. K. Halliday y J. R. Martin, Eds., Writing science: literacy and discursive power. Londres: Taylor and Francis. 223-241.

. 2004. "Grammatical structure: what do we mean?". En Caroline Coffin, Anne Hewings y Kieran O'Halloran, Eds., Applying English Grammar: functional and corpus approaches. Londres: Routledge. 57-76.

Martin, J. R. y David Rose. 2007. Working with discourse: meaning beyond the clause. 2da ed. Londres: Continuum. . 2008. Genre relations: mapping culture. Londres: Equinox.

Martin, J. R. y Peter White. 2005. The language of evaluation: appraisal in English. Basingstoke: Palgrave Macmillan.

Matthiessen, C. M. I. M. 2015. "Halliday on language". En Jonathan Webster, Ed., The Bloomsbury Companion to M. A. K. Halliday. Londres: Bloomsbury. 137-202.

Matthiessen, C. M. I. M. y M. A. K. Halliday. 2009. Systemic functional grammar: a first step into the theory. Beijing: Higher Education Press.

Picallo, M. Carme y Gemma Rigau. 1999. "El posesivo y las relaciones posesivas”. En Ignacio Bosque y Violeta Demonte, Eds., Gramática descriptiva de la lengua española, Vol. 1. Madrid: Espasa-Calpe. 973-1023.

Quiroz, Beatriz. 2015. "La cláusula como movimiento interactivo: una perspectiva semántico-discursiva de la gramática interpersonal del español". Documentação de Estudios em Linguistica Teorica e Aplicada - DELTA, 31.1: 261-301. doi: http://dx.doi. org/10.1590/0102-445023762456121953

. 2016. "Convenciones de notación sistémica". Onomázein 33.2: 412-426. doi: 10.7764/onomazein.33.24

. 2017a. "Gramática interpersonal básica del español: una caracterización sistémicofuncional del sistema de MODO”. Lenguas Modernas 49: 157-182.

. 2017b. "The verbal group”. En Tom Bartlett y Gerard O'Grady, Eds., The Routledge Handbook of Systemic Functional Linguistics. Londres: Routledge. 301-318. 
ESTUDIOS FILOLÓGICOS

Suñer Grataços, Avel-lina. 1999. "La aposición y otras relaciones de predicación en el sintagma nominal”. En Ignacio Bosque y Violeta Demonte, Eds., Gramática Descriptiva de la Lengua Española, Vol. 1, Madrid: Espasa-Calpe. 523-564.

Whorf, Benjamin Lee. 1945. "Grammatical categories". Language 21.1: 1-11. 
